Indranil Biswas*, Tomás L. Gómez, and André Oliveira

\title{
Complex Lagrangians in a hyperKähler manifold and the relative Albanese
}

https://doi.org/10.1515/coma-2020-0106

Received June 29, 2020; accepted October 18, 2020

Abstract: Let $M$ be the moduli space of complex Lagrangian submanifolds of a hyperKähler manifold $X$, and let $\varpi: \widehat{\mathcal{A}} \longrightarrow M$ be the relative Albanese over $M$. We prove that $\widehat{\mathcal{A}}$ has a natural holomorphic symplectic structure. The projection $\varpi$ defines a completely integrable structure on the symplectic manifold $\widehat{\mathcal{A}}$. In particular, the fibers of $\varpi$ are complex Lagrangians with respect to the symplectic form on $\widehat{\mathcal{A}}$. We also prove analogous results for the relative Picard over $M$.

Keywords: HyperKähler manifold, complex Lagrangian, integrable system, Liouville form, Albanese

MSC: 14J42, 53D12, 37K10, 14D21

\section{Introduction}

A compact Kähler manifold admits a holomorphic symplectic form if and only if it admits a hyperKähler structure [1], [10]. To explain this, let $X$ be a compact manifold equipped with almost complex structures $J_{1}, J_{2}, J_{3}$, and let $g$ be a Riemannian metric on $X$, such that $\left(X, J_{1}, J_{2}, J_{3}, g\right)$ is a hyperKähler manifold. Then $g$ defines a $C^{\infty}$ isomorphism,

$$
T^{0,1} X \stackrel{g_{1}}{\longrightarrow}\left(T^{1,0} X\right)^{\star},
$$

where $T^{\mathbb{R}} X \otimes \mathbb{C}=T^{1,0} X \oplus T^{0,1} X$ is the type decomposition with respect to the almost complex structure $J_{1}$; also $J_{2}$ produces a $C^{\infty}$ isomorphism

$$
T^{1,0} X \stackrel{J_{2}^{\prime}}{\longrightarrow} T^{0,1} X
$$

The composition of homomorphisms

$$
T^{1,0} X \stackrel{J_{2}^{\prime}}{\longrightarrow} T^{0,1} X \stackrel{g_{1}}{\longrightarrow}\left(T^{1,0} X\right)^{\star},
$$

which is a section of $\left(T^{1,0} X\right)^{\star} \otimes\left(T^{1,0} X\right)^{\star}$, is actually is a holomorphic symplectic form on the compact Kähler manifold $\left(X, J_{1}, g\right)$. The compact Kähler manifold $\left(X, J_{1}, g\right)$ is Ricci-flat. Conversely, if a compact Kähler manifold admits a holomorphic symplectic form, then its canonical line bundle is holomorphically trivial and hence it admits a Ricci-flat Kähler metric [10]. Let $\left(X, J_{1}, g\right)$ be a Ricci-flat compact Kähler manifold equipped with a holomorphic symplectic form. Then we may recover $J_{2}$ by reversing the above construction. Finally, we have $J_{3}=J_{1} \circ J_{2}$.

\footnotetext{
${ }^{\star}$ Corresponding Author: Indranil Biswas: School of Mathematics, Tata Institute of Fundamental Research, Homi Bhabha Road, Mumbai 400005, India, E-mail: indranil@math.tifr.res.in

Tomás L. Gómez: Instituto de Ciencias Matemáticas (CSIC-UAM-UC3M-UCM), Nicolás Cabrera 15, Campus Cantoblanco UAM, 28049 Madrid, Spain, E-mail: tomas.gomez@icmat.es

André Oliveira: Centro de Matemática da Universidade do Porto (CMUP), Faculdade de Ciências, Rua do Campo Alegre, 687, 4169-007 Porto, Portugal

On leave from: Departamento de Matemática, Universidade de Trás-os-Montes e Alto Douro, UTAD, Quinta dos Prados, 5000-911 Vila Real, Portugal, E-mail: andre.oliveira@fc.up.pt / agoliv@utad.pt
} 
Let $X$ be a compact Kähler Ricci-flat manifold admitting a holomorphic symplectic form. Fix a Ricciflat Kähler form $\omega$ on $X$ (such a Kähler form exists [10]), and take a holomorphic symplectic form $\Phi$ on $X$. It is known that $\Phi$ is parallel (meaning, covariant constant) with respect to the Levi-Civita connection on $X$ associated to $\omega$ [1, p. 760, "Principe de Bochner"], [9, p. 142].

Let $M$ denote the moduli space of compact complex submanifolds of $X$ that are Lagrangian with respect to the symplectic form $\Phi$. Consider the corresponding universal family of Lagrangians

$$
Z \longrightarrow M \text {. }
$$

Let

$$
\varpi: \widehat{\mathcal{A}} \longrightarrow M
$$

be the relative Albanese over $M$. So for any Lagrangian $L \in M$, the fiber of $\widehat{\mathcal{A}}$ over $L$ is $\operatorname{Alb}(L)=$ $H^{0}\left(L, \Omega_{L}^{1}\right)^{\star} / H_{1}(L, \mathbb{Z})$. This $\varphi$ is a holomorphic family of compact complex tori over $M$.

We prove the following (see Theorem 3.3):

The complex manifold $\widehat{\mathcal{A}}$ has a natural holomorphic symplectic form.

The symplectic form on $\widehat{\mathcal{A}}$ is constructed using the canonical Liouville symplectic form on the holomorphic cotangent bundle $T^{\star} M$ of $M$. The symplectic form $\Phi$ on $X$ is implicitly used in the construction of the symplectic form on $\widehat{\mathcal{A}}$. Recall that the Lagrangian submanifolds, and hence $M$, are defined using $\Phi$.

We prove the following (see Lemma 3.4):

The projection $\varpi: \widehat{\mathcal{A}} \longrightarrow M$ defines a completely integrable structure on the symplectic manifold $\widehat{\mathcal{A}}$. In particular, the fibers of $\varpi$ are Lagrangians with respect to the symplectic form on $\widehat{\mathcal{A}}$.

In Section 4 we consider the relative Picard bundle over $M$ for the family $z$ in (1.1). Let

$$
\varpi_{0}: \mathcal{A} \longrightarrow M
$$

be the relative Picard bundle for the family $z$.

We prove that $\mathcal{A}$ is equipped with a natural holomorphic symplectic structure; see Proposition 4.3.

Let $\Theta_{\mathcal{A}}$ denote the above mentioned holomorphic symplectic structure on $\mathcal{A}$. The following lemma is proved (see Lemma 4.4):

The projection $\varpi_{0}: \mathcal{A} \longrightarrow M$ defines a completely integrable structure on $\mathcal{A}$ for the symplectic form $\Theta_{\mathcal{A}}$.

These results are natural generalizations of some known cases of integrable systems, such as the Hitchin system [4] or the Mukai system [7] (see also [2]). One of our motivations has been mirror symmetry; we hope to come back to the study of $\mathcal{A}$ and $\widehat{\mathcal{A}}$ from the point of view of hyperKähler geometry.

\section{Cotangent bundle of family of Lagrangians}

Let $X$ be a compact Kähler Ricci-flat manifold of complex dimension $2 d$ equipped with a Kähler form $\omega$. Let $\Phi$ be a holomorphic symplectic form on $X$ which is parallel with respect to the Levi-Civita connection on $X$ given by the Kähler metric on $X$ associated to $\omega$.

A complex Lagrangian submanifold of $X$ is a compact complex submanifold $L \subset X$ of complex dimension $d$ such that $\iota^{*} \Phi=0$, where

$$
\iota: L \hookrightarrow X
$$

is the inclusion map.

It is known that the infinitesimal deformations of a complex Lagrangian submanifold of $X$ are unobstructed [6], [8], [5]. Furthermore, the moduli space of complex Lagrangian submanifolds of $X$ is a special Kähler manifold [5, p. 84, Theorem 3].

Let $M$ be the moduli space of complex Lagrangian submanifolds of $X$. Let

$$
L \subset X
$$


be a complex Lagrangian submanifold. The point of $M$ representing $L$ will also be denoted by $L$. Let $N_{L} \longrightarrow L$ be the normal bundle of $L \subset X$; it is a quotient bundle of $\iota^{\star} T X$ of rank $d$, where $\iota$ is the map in (2.1). The infinitesimal deformations of the complex submanifold $L$ are parametrized by $H^{0}\left(L, N_{L}\right)$. Since $L$ is complex Lagrangian, the holomorphic symplectic form $\Phi$ on $X$ produces a holomorphic isomorphism

$$
N_{L} \stackrel{\sim}{\longrightarrow}(T L)^{\star}=\Omega_{L}^{1},
$$

where $T L$ (respectively, $\Omega_{L}^{1}$ ) is the holomorphic tangent (respectively, cotangent) bundle of $L$. Using this isomorphism we have

$$
H^{0}\left(L, N_{L}\right)=H^{0}\left(L, \Omega_{L}^{1}\right) .
$$

Among the infinitesimal deformations of the complex submanifold $L$, there are those which arise from deformations within the category of complex Lagrangian submanifolds, meaning those arise from deformations of complex Lagrangian submanifolds as Lagrangian submanifolds. An infinitesimal deformation

$$
\alpha \in H^{0}\left(L, N_{L}\right)=H^{0}\left(L, \Omega_{L}^{1}\right)
$$

of $L$ lies in this subclass if and only if the holomorphic 1 -form $\alpha$ on $L$ is closed [5, pp. 78-79]. But any holomorphic 1-form on $L$ is closed because $L$ is Kähler. Therefore, $M$ is in fact an open subset of the corresponding Douady space for $X$.

Consider the Ricci-flat Kähler form $\omega$ on $X$. The form

$$
\omega_{L}:=i^{\star} \omega
$$

on $L$ is also Kähler, where $\iota$ is the map in (2.1). Therefore, the pairing

$$
\phi_{L}: H^{0}\left(L, \Omega_{L}^{1}\right) \otimes H^{1}\left(L, \mathcal{O}_{L}\right) \longrightarrow \mathbb{C}, w \otimes c \longmapsto \int_{L} w \wedge c \wedge \omega_{L}^{d-1}
$$

is nondegenerate. We shall identify $H^{1}\left(L, \mathcal{O}_{L}\right)$ with $H^{0}\left(L, \Omega_{L}^{1}\right)^{\star}$ using this nondegenerate pairing.

It was noted above that

$$
T_{L} M=H^{0}\left(L, N_{L}\right)=H^{0}\left(L, \Omega_{L}^{1}\right) .
$$

Using the pairing in (2.3) and (2.4), we have

$$
T_{L}^{\star} M=H^{0}\left(L, \Omega_{L}^{1}\right)^{\star}=H^{1}\left(L, \mathcal{O}_{L}\right) .
$$

Let

$$
z \subset X \times M
$$

be the universal family of complex Lagrangians over $M$. So $z$ is the locus of all $\left(x, L^{\prime}\right) \in X \times M$ such that $x \in L^{\prime} \subset X$. Consider the natural projections $p_{X}: X \times M \longrightarrow X$ and $p_{M}: X \times M \longrightarrow M$. Let

$$
p: z \longrightarrow X \text { and } q: z \longrightarrow M
$$

be the restrictions of $p_{X}$ and $p_{M}$ respectively to the submanifold $z \subset X \times M$. So $p\left(q^{-1}\left(L^{\prime}\right)\right) \subset X$ for every $L^{\prime} \in M$ is the Lagrangian $L^{\prime}$ itself.

Let $\Omega_{z / M}^{1} \longrightarrow Z$ be the relative cotangent bundle for the projection $q$ to $M$ in (2.7). It fits in the short exact sequence of holomorphic vector bundles

$$
0 \longrightarrow q^{\star} \Omega_{M}^{1} \longrightarrow \Omega_{\mathcal{Z}}^{1} \longrightarrow \Omega_{\mathcal{Z} / M}^{1} \longrightarrow 0
$$

over $M$. The direct image $R^{1} q_{\star} \mathrm{O}_{z}$ fits in the short exact sequence of sheaves on $M$

$$
0 \longrightarrow R^{0} q_{\star} \Omega_{\mathcal{Z} / M}^{1} \longrightarrow R^{1} q_{\star} \underline{\mathbb{C}} \longrightarrow R^{1} q_{\star} \mathcal{O}_{Z} \longrightarrow 0,
$$


where $\underline{\mathbb{C}}$ is the constant sheaf on $z$ with stalk $\mathbb{C}$. The direct image $R^{1} q_{\star} \mathbb{\mathbb { C }}$ is a flat complex vector bundle, equipped with the Gauss-Manin connection. We briefly recall the construction of the Gauss-Manin connection. For any point $L^{\prime}=y \in M$, let $U_{y} \subset M$ be a contractible open neighborhood of $y$. Since $U_{y}$ is contractible, the inverse image $q^{-1}\left(U_{y}\right)$ is diffeomorphic to $U_{y} \times L^{\prime}$ such that the diffeomorphism between $q^{-1}\left(U_{y}\right)$ and $U_{y} \times L^{\prime}$ takes $q$ to the natural projection from $U_{y} \times L^{\prime}$ to $U_{y}$. Using this diffeomorphism, the restriction of $R^{1} q * \mathbb{C}$ to $U_{y}$ coincides with the trivial vector bundle

$$
U_{y} \times H^{1}\left(L^{\prime}, \mathbb{C}\right) \longrightarrow U_{y}
$$

with fiber $H^{1}\left(L^{\prime}, \mathbb{C}\right)$. Using this isomorphism between $\left.\left(R^{1} q_{\star} \mathbb{C}\right)\right|_{U_{y}}$ and the trivial vector bundle $U_{y} \times H^{1}\left(L^{\prime}, \mathbb{C}\right)$ in (2.9), the trivial connection on the trivial vector bundle in (2.9) produces a flat connection on $\left.\left(R^{1} q_{\star} \mathbb{\mathbb { C }}\right)\right|_{U_{y}}$. This connection on $\left.\left(R^{1} q * \underline{\mathbb{C}}\right)\right|_{U_{y}}$ does not depend on the choice of the diffeomorphism between $q^{-1}\left(U_{y}\right)$ and $U_{y} \times L^{\prime}$. Consequently, these locally defined flat connections on $R^{1} q_{\star} \mathbb{C}$ patch together compatibly to define a flat connection on $R^{1} q * \mathbb{C}$. This flat connection is the Gauss-Manin connection mentioned above.

Since the Gauss-Manin connection on $R^{1} q_{\star} \mathbb{\mathbb { C }}$ is flat, and $M$ is a complex manifold, the Gauss-Manin connection produces a natural holomorphic structure on the $C^{\infty}$ vector bundle $R^{1} q_{\star} \mathbb{\mathbb { C }}$. The direct image $R^{0} q_{\star} \Omega_{z / M}^{1}$ is a holomorphic subbundle of $R^{1} q_{\star} \mathbb{C}$, but it is not preserved by the flat connection in general. The holomorphic structure on the quotient $R^{1} q_{\star} \mathcal{O}_{z}$ induced by that of $R^{1} q \star \mathbb{C}$ coincides with its own holomorphic structure; the fiber of $R^{1} q_{*} \mathcal{O}_{z}$ over any $L^{\prime} \in M$ is $H^{1}\left(L^{\prime}, \mathcal{O}_{L^{\prime}}\right)$.

Since $\omega_{L}$ in (2.2) is the restriction of a global Kähler form on $X$, the section of $R^{2} q_{\star} \underline{\mathbb{C}}$

$$
M \longrightarrow R^{2} q \star \underline{\mathbb{C}}, L^{\prime} \longmapsto\left[\omega_{L^{\prime}}\right]=\left[\left.\omega\right|_{L^{\prime}}\right] \in H^{2}\left(L^{\prime}, \mathbb{C}\right)
$$

is covariant constant with respect to the Gauss-Manin connection on $R^{2} q \star \mathbb{C}$. Consequently, the homomorphism

$$
\left(R^{1} q \star \underline{\mathbb{C}}\right) \otimes\left(R^{1} q \star \underline{\mathbb{C}}\right) \longrightarrow \mathbb{C},
$$

that sends any $v \otimes w \in\left(R^{1} q_{\star} \underline{\mathbb{C}}\right)_{t} \otimes\left(R^{1} q_{\star} \underline{\mathbb{C}}\right)_{t}, t \in M$, to

$$
\int_{q^{-1}(t)} v \wedge w \wedge\left(\left.\omega\right|_{q^{-1}(t)}\right)^{d-1} \in \mathbb{C}
$$

is also covariant constant with respect to the connection on $\left(R^{1} q_{\star} \mathbb{C}\right) \otimes\left(R^{1} q_{\star} \mathbb{C}\right)$ induced by the Gauss-Manin connection on $R^{1} q * \mathbb{C}$. Hence the pairing in (2.10) produces a holomorphic isomorphism of vector bundles

$$
\left(q_{\star} \Omega_{Z / M}^{1}\right)^{*} \stackrel{\sim}{\longrightarrow} R^{1} q_{\star} \mathcal{O}_{Z}
$$

on $M$. The restriction of this isomorphism to any point $L \in M$ coincides with the isomorphism $H^{0}\left(L, \Omega_{L}^{1}\right)^{\star}=$ $H^{1}\left(L, \mathcal{O}_{L}\right)$ in $(2.5)$.

On the other hand, the pointwise isomorphisms in (2.4) combine together to produce a holomorphic isomorphism of vector bundles

$$
\Omega_{M}^{1} \stackrel{\sim}{\longrightarrow}\left(q_{\star} \Omega_{\mathcal{Z} / M}^{1}\right)^{*}
$$

on $M$. Composing the isomorphisms in (2.11) and (2.12), we obtain a holomorphic isomorphism of vector bundles

$$
\chi: \Omega_{M}^{1} \longrightarrow R^{1} q_{\star} \mathcal{O}_{z}
$$

over $M$.

For notational convenience, the total space of $R^{1} q_{\star} \mathcal{O}_{z}$ will be denoted by $y$. Let

$$
\gamma: y \longrightarrow M
$$

be the natural projection. Consider the canonical Liouville 1-form on $\Omega_{M}^{1}$. Using the isomorphism $\chi$ in (2.13) this Liouville 1-form on $\Omega_{M}^{1}$ gives a holomorphic 1-form on $y$. Let

$$
\theta \in H^{0}\left(y, \Omega_{y}^{1}\right)
$$


be the holomorphic 1-form given by the Liouville 1-form on $\Omega_{M}^{1}$. Note that for any $L \in M$, and any $v \in \gamma^{-1}(L)$, the form

$$
\theta(v): T_{v} y \longrightarrow \mathbb{C}
$$

coincides with the composition of homomorphisms

$$
T_{v} y \stackrel{d \gamma}{\longrightarrow} T_{L} M=H^{0}\left(L, \Omega_{L}^{1}\right) \stackrel{\phi_{L}(-, v)}{\longrightarrow} \mathbb{C},
$$

where $\phi_{L}$ is the bilinear pairing constructed in (2.3), and $d \gamma$ is the differential of the projection $\gamma$ in (2.14); the above identification

$$
T_{L} M=H^{0}\left(L, \Omega_{L}^{1}\right)
$$

is the one constructed in (2.4).

It is straight-forward to check that the 2 -form

$$
d \theta \in H^{0}\left(y, \Omega_{y}^{2}\right)
$$

is a holomorphic symplectic form on the manifold $y$ in (2.14). Indeed, $d \theta$ evidently coincides with the 2 -form on $y$ given by the Liouville symplectic form on $\Omega_{M}^{1}$ via the isomorphism $\chi$ in (2.13).

\section{The family of Albanese tori}

Take a compact complex Lagrangian submanifold $L \subset X$ represented by a point of $M$. We know that $T_{L}^{\star} M \cong$ $H^{0}\left(L, \Omega_{L}^{1}\right)^{\star}$ (see (2.4)). Note that the non-degenerate pairing

$$
H^{1,0}(L) \otimes H^{d-1, d}(L) \longrightarrow \mathbb{C}, \alpha \otimes \beta \longmapsto \int_{L} \alpha \wedge \beta,
$$

which is also the Serre duality pairing, yields an isomorphism

$$
H^{0}\left(L, \Omega_{L}^{1}\right)^{\star} \cong H^{d}\left(L, \Omega_{L}^{d-1}\right) .
$$

For a fixed $L$, we have the Hodge decomposition

$$
H^{2 d-1}(L, \mathbb{C})=H^{d, d-1}(L) \oplus H^{d-1, d}(L) ;
$$

but if we move $L$ in the family $M$, meaning if we consider the universal family

$$
q: Z \longrightarrow M
$$

in (2.6), then only $R^{d-1} q_{\star} \Omega_{\mathcal{Z} / M}^{d}$ is a holomorphic subbundle of $R^{2 d-1} q_{\star} \underline{\mathbb{C}}$, and we have the short exact sequence of holomorphic vector bundles

$$
0 \longrightarrow R^{d-1} q_{\star} \Omega_{\mathcal{Z} / M}^{d} \longrightarrow R^{2 d-1} q_{\star} \underline{\mathbb{C}} \longrightarrow R^{d} q_{\star} \Omega_{\mathcal{Z} / M}^{d-1} \longrightarrow 0,
$$

on $M$.

The holomorphic vector bundle $R^{2 d-1} q_{\star} \underline{\mathbb{C}}$ is equipped with the Gauss-Manin connection, which is an integrable connection. The quotient $R^{d} q_{\star} \Omega_{z / M}^{d-1}$, in (3.1), of $R^{2 d-1} q \star \underline{\mathbb{C}}$ is a holomorphic vector bundle on $M$ with fiber $H^{d}\left(L^{\prime}, \Omega_{L^{\prime}}^{d-1}\right)$ over any $L^{\prime} \in M$.

The homomorphism

$$
\left(R^{1} q_{\star} \mathbb{\mathbb { C }}\right) \otimes\left(R^{2 d-1} q_{\star} \underline{\mathbb{C}}\right) \longrightarrow \underline{\mathbb{C}},
$$

that sends any $\alpha \otimes \beta \in\left(R^{1} q \star \underline{\mathbb{C}}\right)_{t} \otimes\left(R^{2 d-1} q \star \underline{\mathbb{C}}\right)_{t}, t \in M$, to

$$
\int_{q^{-1}(t)} v \wedge w \in \mathbb{C}
$$


is covariant constant with respect to the connection on $\left(R^{1} q * \underline{\mathbb{C}}\right) \otimes\left(R^{2 d-1} q_{\star} \underline{\mathbb{C}}\right)$ induced by the Gauss-Manin connections on $R^{1} q \star \underline{\mathbb{C}}$ and $R^{2 d-1} q \star \underline{\mathbb{C}}$. Consequently, the pairing in (3.2) yields a holomorphic isomorphism of vector bundles

$$
\left(q_{\star} \Omega_{z / M}^{1}\right)^{\star} \stackrel{\sim}{\longrightarrow} R^{d} q_{\star} \Omega_{Z / M}^{d-1}
$$

over $M$. Combining this isomorphism with the isomorphism in (2.12) we get a holomorphic isomorphism of vector bundles

$$
\widehat{\chi}: \Omega_{M}^{1} \stackrel{\sim}{\longrightarrow} R^{d} q \star \Omega_{Z / M}^{d-1}
$$

over $M$.

We shall denote the total space of the holomorphic vector bundle $R^{d} q_{\star} \Omega_{\mathcal{Z} / M}^{d-1}$ by $\mathcal{W}$, so

$$
\mathcal{W}:=R^{d} q_{\star} \Omega_{\mathcal{Z} / M}^{d-1} \stackrel{\widehat{\gamma}}{\longrightarrow} M
$$

is a holomorphic fiber bundle.

As in Section 2, consider the canonical Liouville holomorphic 1-form on the total space of $\Omega_{M}^{1}$. Using the isomorphism in (3.3), this Liouville 1 -form on the total space of $\Omega_{M}^{1}$ produces a holomorphic 1 -form on $\mathcal{W}$ in (3.4). Let

$$
\theta^{\prime} \in H^{0}\left(\mathcal{W}, \Omega_{\mathcal{W}}^{1}\right)
$$

be this holomorphic 1 -form on $\mathcal{W}$. We note that

$$
d \theta^{\prime} \in H^{0}\left(\mathcal{W}, \Omega_{\mathcal{W}}^{2}\right)
$$

is a holomorphic symplectic form on $\mathcal{W}$. Indeed, the isomorphism in (3.3) takes $d \theta^{\prime}$ to the Liouville symplectic form on the total space of $\Omega_{M}^{1}$.

Remark 3.1. Note that while the Kähler form $\omega$ on $X$ was used in the construction of the symplectic form $d \theta$ on $y$ in (2.16) (see the pairing in (2.10)), the construction of $d \theta^{\prime}$ in (3.5) does not use the Kähler form $\omega$ on $X$. We recall that the isomorphism in (2.12) is constructed from the the pointwise isomorphisms in (2.4). Note that the isomorphism in (2.4) does not depend on the Kähler form $\omega$.

The Albanese $\operatorname{Alb}(Y)$ of a compact Kähler manifold $Y$ is defined to be

$$
\operatorname{Alb}(Y)=H^{0}\left(Y, \Omega_{Y}^{1}\right)^{\star} / H_{1}(Y, \mathbb{Z})=H^{n}\left(Y, \Omega_{Y}^{n-1}\right) / H_{1}(Y, \mathbb{Z}),
$$

where $n=\operatorname{dim}_{\mathbb{C}} Y$ (see [3, p. 331]). It is a compact complex torus.

For each point $L \in M$, consider the composition of homomorphisms

$$
H^{2 d-1}(L, \mathbb{Z}) \longrightarrow H^{2 d-1}(L, \mathbb{C}) \longrightarrow H^{2 d-1}(L, \mathbb{C}) / H^{d-1}\left(L, \Omega_{L}^{d}\right)=H^{d}\left(L, \Omega_{L}^{d-1}\right)
$$

(see (3.1)). It produces a homomorphism

$$
R^{2 d-1} q \star \underline{\mathbb{Z}} \longrightarrow \mathcal{W}
$$

where $\mathcal{W}$ is defined in (3.4).

Remark 3.2. The Gauss-Manin connection on $R^{2 d-1} q_{\star} \underline{\mathbb{C}} \longrightarrow M$ evidently preserves the subbundle of lattices

$$
R^{2 d-1} q * \underline{\mathbb{Z}} \subset R^{2 d-1} q \star \underline{\mathbb{C}} .
$$

From this it follows immediately that the $C^{\infty}$ submanifold $R^{2 d-1} q \star \underline{\mathbb{Z}} \subset \mathcal{W}$ in (3.6) is in fact a complex submanifold.

The quotient

$$
\widehat{\mathcal{A}}:=\mathcal{W} /\left(R^{2 d-1} q * \underline{\mathbb{Z}}\right) \longrightarrow M
$$


for the homomorphism in (3.6) is in fact a holomorphic family of compact complex tori over $M$. Note that the fiber of $\widehat{\mathcal{A}}$ over each $L \in M$ is the Albanese torus

$$
\operatorname{Alb}(L)=H^{d}\left(L, \Omega_{L}^{d-1}\right) / H^{2 d-1}(L, \mathbb{Z}) .
$$

For any complex Lagrangian $L \in M$, using Serre duality,

$$
H^{d}\left(L, \Omega_{L}^{d-1}\right)=H^{0}\left(L, \Omega_{L}^{1}\right)^{\star},
$$

and the underlying real vector space for $H^{0}\left(L, \Omega_{L}^{1}\right)^{\star}$ is identified with

$$
H^{1}(L, \mathbb{R})^{\star}=H_{1}(L, \mathbb{R}) .
$$

Using Poincaré duality for $L$, we have $H^{2 d-1}(L, \mathbb{Z}) /$ Torsion $=H_{1}(L, \mathbb{Z}) /$ Torsion. Consequently, $\widehat{\mathcal{A}}$ in (3.7) admits the following isomorphism:

$$
\widehat{\mathcal{A}}=\mathcal{W} /\left(R^{2 d-1} q_{\star} \mathbb{Z}\right)=\mathcal{W} / \widetilde{H}_{1}(\mathbb{Z})=\left(q_{\star} \Omega_{\mathcal{Z} / M}^{1}\right)^{\star} / \widetilde{H}_{1}(\mathbb{Z}),
$$

where $\widetilde{H}_{1}(\mathbb{Z})$ is the local system on $M$ whose stalk over any $L \in M$ is $H_{1}(L, \mathbb{Z}) /$ Torsion. Also we have the isomorphism of real tori

$$
\widehat{\mathcal{A}}=\left(R^{1} q \star \underline{\mathbb{R}}\right)^{\star} / \widetilde{H}_{1}(\mathbb{Z})=\widetilde{H}_{1}(\mathbb{R}) / \widetilde{H}_{1}(\mathbb{Z}),
$$

where $\widetilde{H}_{1}(\mathbb{R})$ is the local system on $M$ whose stalk over any $L \in M$ is $H_{1}(L, \mathbb{R})$.

Theorem 3.3. The 2-form $d \theta^{\prime}$ in (3.5) on $\mathcal{W}$ descends to the quotient torus $\widehat{\mathcal{A}}$ in (3.7).

Proof. Take a point $L_{0} \in M$. In [5] Hitchin constructed a $C^{\infty}$ coordinate function on $M$ defined around the point $L_{0} \in M$ that takes values in $H^{1}\left(L_{0}, \mathbb{R}\right)$ [5, p. 79, Theorem 2]; we will briefly recall this construction.

Take any

$$
c \in H_{1}\left(L_{0}, \mathbb{Z}\right) / \text { Torsion . }
$$

Let $U \subset M$ be a contractible neighborhood of the point $L_{0}$. Choose a $S^{1}$-subbundle of the fiber bundle $z$ (see (2.6)) over $U$

$$
\left.B \stackrel{I_{U}}{\longrightarrow} Z\right|_{U} \stackrel{q}{\longrightarrow} U
$$

such that the fiber of the $S^{1}$-bundle $B$ over $L_{0}$ represents the homology class $c$ in (3.10); recall that the fiber of $Z$ over the point $L_{0} \in M$ is the Lagrangian $L_{0}$ itself.

Consider the symplectic form $\Phi$ on $X$. Integrating $\iota_{U}^{\star} p^{*} \operatorname{Re}(\Phi)$ along the fibers of $B$, where $\iota_{U}$ and $p$ are the maps in (3.11) and (2.7) respectively, we get a closed 1-form $\xi_{c}$ on $U$. Let $f_{c}$ be the unique function on $U$ such that $f_{c}\left(L_{0}\right)=0$ and $d f_{c}=\xi_{c}$. Now, let

$$
\mu: U \longrightarrow H^{1}\left(L_{0}, \mathbb{R}\right)
$$

be the function uniquely determined by the condition that $\phi_{L_{x}}(c \otimes \mu(x))=f_{c}(x)$ for all $x \in U$ and $c \in$ $H_{1}\left(L_{0}, \mathbb{Z}\right) /$ Torsion, where $\phi_{L_{x}}$ is the pairing constructed as in (2.3) for the complex Lagrangian $L_{x}=q^{-1}(x) \subset$ $X$, where $q$ is the projection in (2.7). This $\mu$ is a local diffeomorphism [5, p. 79, Theorem 2].

Using the Kähler form $\omega_{L_{0}}:=\left.\omega\right|_{L_{0}}$ on $L_{0}$ (see (2.2)), we identify $H^{1}\left(L_{0}, \mathbb{R}\right)$ with $H_{1}\left(L_{0}, \mathbb{R}\right)$ as follows. Since the pairing

$$
H^{1}\left(L_{0}, \mathbb{R}\right) \otimes H^{1}\left(L_{0}, \mathbb{R}\right) \longrightarrow \mathbb{R}, v \otimes w \longmapsto \int_{L} v \wedge w \wedge \omega_{L_{0}}^{d-1}
$$

is nondegenerate, it produces an isomorphism

$$
H^{1}\left(L_{0}, \mathbb{R}\right) \stackrel{\sim}{\longrightarrow} H^{1}\left(L_{0}, \mathbb{R}\right)^{*}=H_{1}\left(L_{0}, \mathbb{R}\right) .
$$

On the other hand, there is the natural homomorphism $H^{1}\left(L_{0}, \mathbb{Z}\right) \longrightarrow H^{1}\left(L_{0}, \mathbb{R}\right)$. Let

$$
\Gamma \subset H_{1}\left(L_{0}, \mathbb{R}\right)
$$


be the subgroup that corresponds to $H^{1}\left(L_{0}, \mathbb{Z}\right)$ by the isomorphism in (3.12).

Using the above coordinate function $\mu$ on $U$, we have

$$
\mathcal{T}^{\star} U \stackrel{\sim}{\longrightarrow} \mathcal{T}^{\star} \mu(U)=\mu(U) \times H^{1}\left(L_{0}, \mathbb{R}\right)^{\star}=\mu(U) \times H_{1}\left(L_{0}, \mathbb{R}\right),
$$

where $\mathcal{T}^{\star}$ denotes the real cotangent bundle.

The Liouville symplectic form on $\mathcal{T}^{\star} \mu(U)$ is clearly the constant 2 -form on

$$
H^{1}\left(L_{0}, \mathbb{R}\right) \times H_{1}\left(L_{0}, \mathbb{R}\right)
$$

given by the natural isomorphism of $H_{1}\left(L_{0}, \mathbb{R}\right)$ with $H^{1}\left(L_{0}, \mathbb{R}\right)^{\star}$. From this it follows immediately that

$$
\mu(U) \times \Gamma \subset \mu(U) \times H_{1}\left(L_{0}, \mathbb{R}\right)
$$

is a Lagrangian submanifold with respect to the Liouville symplectic form on $\mathcal{T}^{*} \mu(U)$, where $\Gamma$ defined in (3.13).

Since $\mu(U) \times \Gamma \subset \mu(U) \times H_{1}\left(L_{0}, \mathbb{R}\right)$ is a Lagrangian submanifold, it follows that for $\mathcal{W}=\left(R^{1} q \star \mathbb{R}\right)^{\star}$ (see (3.9) and (3.4)), the image of the natural map

$$
\widetilde{H}_{1}(\mathbb{Z}) \longrightarrow\left(R^{1} q * \underline{\mathbb{R}}\right)^{\star}=\mathcal{W}
$$

(see (3.8)) is Lagrangian with respect to the real symplectic form $\operatorname{Re}\left(d \theta^{\prime}\right)$ on $\mathcal{W}$, where $d \theta^{\prime}$ is constructed in (3.5).

It was noted in Remark 3.2 that $R^{2 d-1} q \star \underline{\mathbb{Z}}$ is a complex submanifold of $\mathcal{W}$. Consequently, the 2-form on $R^{2 d-1} q \star \underline{\mathbb{Z}}$ obtained by restricting the holomorphic 2-form $d \theta^{\prime}$ on $\mathcal{W}$ is also holomorphic. Since the real part of the holomorphic 2-from on $R^{2 d-1} q * \underline{\mathbb{Z}}$ given by $d \theta^{\prime}$ vanishes identically, we conclude that the holomorphic 2from on $R^{2 d-1} q * \underline{\mathbb{Z}}$, given by $d \theta^{\prime}$, itself vanishes identically. Therefore, $R^{2 d-1} q * \underline{\mathbb{Z}}$ is a Lagrangian submanifold of the holomorphic symplectic manifold $\mathcal{W}$ equipped with the holomorphic symplectic form $d \theta^{\prime}$.

To complete the proof we recall a general property of the Liouville symplectic form.

Let $N$ be a manifold and $\alpha$ a 1 -form on $N$. Let

$$
t: T^{\star} N \longrightarrow T^{\star} N
$$

be the diffeomorphism that sends any $v \in T_{n}^{\star} N$ to $v+\alpha(n)$. If $\psi$ is the Liouville symplectic form on $T^{\star} N$, the

$$
t^{\star} \psi=\psi+d \alpha .
$$

In particular, the map $t$ preserves $\psi$ if and only if the form $\alpha$ is closed. Also, the image of $t$ is Lagrangian submanifold of $T^{\star} N$ for $\psi$ if and only $\alpha$ is closed.

Since

$$
R^{2 d-1} q \star \underline{\mathbb{Z}}
$$

is a Lagrangian submanifold of the symplectic manifold $\left(\mathcal{W}, d \theta^{\prime}\right)$, from the above property of the Liouville symplectic form it follows immediately that the 2 -form $d \theta^{\prime}$ on $\mathcal{W}$ descends to the quotient space $\widehat{\mathcal{A}}$ in (3.7).

Let

$$
q_{\widehat{\mathcal{A}}}: \mathcal{W} \longrightarrow \widehat{\mathcal{A}}:=\mathcal{W} /\left(R^{2 d-1} q \star \underline{\mathbb{Z}}\right)
$$

be the quotient map (see (3.7)). From Theorem 3.3 we know that there is a unique 2 -form

$$
\Theta_{\widehat{\mathcal{A}}} \in H^{0}\left(\widehat{\mathcal{A}}, \Omega_{\widehat{\mathcal{A}}}^{2}\right)
$$

such that

$$
q_{\widehat{\mathcal{A}}}^{\star} \Theta_{\widehat{\mathcal{A}}}=d \theta^{\prime},
$$

where $q_{\mathcal{A}}$ is the map in (3.14). Since $d \theta^{\prime}$ is a holomorphic symplectic form, it follows immediately that $\Theta_{\widehat{\mathcal{A}}}$ is a holomorphic symplectic form on $\widehat{\mathcal{A}}$. 
The projection $\widehat{\gamma}: \mathcal{W} \longrightarrow M$ in (3.4) clearly descends to a map from $\mathcal{A}$ to $M$. Let

$$
\varpi: \widehat{\mathcal{A}} \longrightarrow M
$$

be the map given by $\widehat{\gamma}$; so we have

$$
\widehat{\gamma}=\varpi \circ q_{\widehat{\mathcal{A}}},
$$

where $q_{\widehat{\mathcal{A}}}$ is constructed in (3.14).

Lemma 3.4. The projection $\varpi$ in (3.17) defines a completely integrable structure on $\widehat{\mathcal{A}}$ for the symplectic form $\Theta_{\widehat{\mathcal{A}}}$ constructed in (3.15). In particular, the fibers of $\varpi$ are Lagrangians with respect to the symplectic form $\Theta_{\widehat{\mathcal{A}}}$.

Proof. Recall that $\mathcal{W}$ is holomorphically identified with $\Omega_{M}^{1}$ by the map $\widehat{\chi}$ in (3.3) (see (3.4)). This map $\widehat{\chi}$ takes the Liouville symplectic form on $\Omega_{M}^{1}$ to the symplectic form $d \theta^{\prime}$ on $\mathcal{W}$. Therefore, from (3.14) and (3.16) we conclude that $\widehat{\mathcal{A}}$ is locally isomorphic to $\Omega_{M}^{1}$ such that the projection $\varpi$ is taken to the natural projection $\Omega_{M}^{1} \longrightarrow M$, and the symplectic form $\Theta_{\widehat{\mathcal{A}}}$ on $\widehat{\mathcal{A}}$ is taken to the Liouville symplectic form on $\Omega_{M}^{1}$. The lemma follows immediately from these, because the natural projection $\Omega_{M}^{1} \longrightarrow M$ defines a completely integrable structure on $\Omega_{M}^{1}$ for the Liouville symplectic form.

\section{The relative Picard group}

For any $L \in M$, consider the homomorphisms

$$
H^{1}(L, \mathbb{Z}) \longrightarrow H^{1}(L, \mathbb{C}) \longrightarrow H^{1}\left(L, \mathcal{O}_{L}\right),
$$

where $H^{1}(L, \mathbb{Z}) \longrightarrow H^{1}(L, \mathbb{C})$ is the natural homomorphism given by the inclusion of $\mathbb{Z}$ in $\mathbb{C}$, and the projection $H^{1}(L, \mathbb{C}) \longrightarrow H^{1}\left(L, \mathcal{O}_{L}\right)$ corresponds to the isomorphism

$$
H^{1}(L, \mathbb{C}) / H^{0}\left(L, \Omega_{L}^{1}\right)=H^{1}\left(L, \mathcal{O}_{L}\right)
$$

(see (2.8)). The image of the composition of homomorphisms in (4.1) is actually a cocompact lattice in $H^{1}\left(L, \mathcal{O}_{L}\right)$; so $H^{1}\left(L, \mathcal{O}_{L}\right) / H^{1}(L, \mathbb{Z})$ is a compact complex torus. We note that the composition of homomorphisms in (4.1) is in fact injective. When the compact complex manifold $L$ is a complex projective variety, then $H^{1}\left(L, \mathcal{O}_{L}\right) / H^{1}(L, \mathbb{Z})$ is in fact an abelian variety.

As $L$ moves over the family $M$, these cocompact lattices fit together to produce a $C^{\infty}$ submanifold of the complex manifold $y$ in (2.14).

The Gauss-Manin connection on $R^{1} q \star \underline{\mathbb{C}} \longrightarrow M$ evidently preserves the above bundle of cocompact lattices $R^{1} q \star \underline{\mathbb{Z}} \subset R^{1} q \star \underline{\mathbb{C}}$. From this it follows immediately that the above $C^{\infty}$ submanifold $R^{1} q \star \mathbb{Z} \subset y$ is in fact a complex submanifold.

Taking fiber-wise quotients, we conclude that

$$
\mathcal{A}:=y /\left(R^{1} q \star \underline{\mathbb{Z}}\right) \longrightarrow M
$$

is a holomorphic family of compact complex tori over $M$.

Remark 4.1. Let $Y$ be a compact Kähler manifold. Consider the short exact sequence of sheaves on $Y$ given by the exponential map

$$
0 \longrightarrow \underline{\mathbb{Z}} \longrightarrow \mathcal{O}_{Y} \stackrel{\lambda \mapsto \exp (2 \pi \sqrt{-1} \lambda)}{\longrightarrow} \mathcal{O}_{Y}^{\star} \longrightarrow 0,
$$

where $\mathcal{O}_{Y}^{\star}$ is a multiplicative sheaf of holomorphic functions with values in $\mathbb{C} \backslash\{0\}$. For the corresponding long exact sequence of cohomologies

$$
H^{1}(Y, \underline{\mathbb{Z}}) \longrightarrow H^{1}\left(Y, \mathcal{O}_{Y}\right) \longrightarrow H^{1}\left(Y, \mathcal{O}_{Y}^{\star}\right) \stackrel{c_{1}}{\longrightarrow} H^{2}(Y, \underline{\mathbb{Z}}),
$$


where the connecting homomorphism $c_{1}$ sends any holomorphic line bundle $\xi \in H^{1}\left(Y, \mathcal{O}_{Y}^{\star}\right)$ to $c_{1}(\xi)$, the quotient

$$
H^{1}\left(Y, \mathcal{O}_{Y}\right) / H^{1}(Y, \underline{\mathbb{Z}})
$$

gets identified with the Picard group $\operatorname{Pic}^{0}(Y)$ that parametrizes the topologically trivial holomorphic line bundles on $Y$.

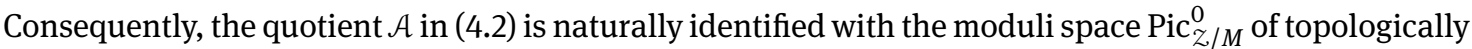
trivial holomorphic line bundles on the fibers of $q: Z \longrightarrow M$. In other words, $\mathcal{A}$ parametrizes all pairs of the form $(L, \xi)$, where $L \in M$, and $\xi$ is a topologically trivial holomorphic line bundle on $L$.

Recall that we have the holomorphic symplectic form $d \theta$ on $y$, where $\theta$ is constructed in (2.15).

Remark 4.2. Recall from Remark 3.1 that $d \theta$ does depend on the Kähler form $\omega$ on $X$.

Proposition 4.3. The 2-form $d \theta$ on $y$ descends to the quotient space $\mathcal{A}$ in (4.2), or in other words, $d \theta$ is the pullback of a 2 -form on $\mathcal{A}$.

Proof. The proof of the proposition is very similar to the proof of Theorem 3.3. As before, the local coordinate functions on $M$ constructed in [5] play a crucial role. We omit the details of the proof.

Let

$$
q_{\mathcal{A}}: y \longrightarrow \mathcal{A}:=y /\left(R^{1} q \star \underline{\mathbb{Z}}\right)
$$

be the quotient map (see (4.2)). Let $\Theta_{\mathcal{A}} \in H^{0}\left(\mathcal{A}, \Omega_{\mathcal{A}}^{2}\right)$ be the holomorphic symplectic form given by Proposition 4.3, so

$$
q_{\mathcal{A}}^{\star} \Theta_{\mathcal{A}}=d \theta,
$$

where $q_{\mathcal{A}}$ is the map in (4.3).

The projection $\gamma$ in (2.14) clearly descends to a map

$$
\varpi_{0}: \mathcal{A} \longrightarrow M
$$

Note that the isomorphism between $\mathcal{A}$ and $\operatorname{Pic}_{\mathcal{Z} / M}^{0}$ in Remark 4.1 takes $\varpi_{0}$ to the forgetful map

$$
\operatorname{Pic}_{z / M}^{0} \longrightarrow M
$$

that forgets the line bundle, or in other words, it sends any $(L, \xi) \in \operatorname{Pic}_{\mathcal{Z} / M}^{0}$ to the complex Lagrangian $L$ forgetting the line bundle $\xi$.

Lemma 4.4. The projection $\varpi_{0}$ in (4.5) defines a completely integrable structure on $\mathcal{A}$ for the symplectic form $\Theta_{\mathcal{A}}$.

Proof. Recall that $y$ is holomorphically identified with $\Omega_{M}^{1}$ by the map $\chi$ in (2.13), and $\chi$ takes the Liouville symplectic form on $\Omega_{M}^{1}$ to the symplectic form $d \theta$ on $y$. Therefore, from (3.14) and (4.4) we conclude that $\mathcal{A}$ is locally isomorphic to $\Omega_{M}^{1}$ such that the projection $\varpi_{0}$ is taken to the natural projection $\Omega_{M}^{1} \longrightarrow M$, and the symplectic form $\Theta_{\mathcal{A}}$ on $\mathcal{A}$ is taken to the Liouville symplectic form on $\Omega_{M}^{1}$. The lemma follows immediately from these.

Acknowledgements: We thank the referee for helpful comments. The first author is partially supported by a J. C. Bose fellowship. The second author is supported by Ministerio de Ciencia e Innovación of Spain (grants MTM2016-79400-P, PID2019-108936GB-C21, and ICMAT Severo Ochoa project SEV-2015-0554) and CSIC (2019AEP151 and Ayuda extraordinaria a Centros de Excelencia Severo Ochoa 20205CEX001) The third author is supported by CMUP, financed by national funds through FCT - Fundação para a Ciência e a Tecnologia, I.P., under the project with reference UIDB/00144/2020. The second and third authors wish to thank TIFR Mumbai for hospitality and for the excellent conditions provided. 


\section{References}

[1] A. Beauville, Variétés Kähleriennes dont la première classe de Chern est nulle, Jour. Differ. Geom. 18 (1983), $755-782$.

[2] R. Donagi, L. Ein and R. K. Lazarsfeld, Nilpotent cones and sheaves on K3 surfaces, Birational algebraic geometry (Baltimore, MD, 1996), 51-61, Contemp. Math., 207, Amer. Math. Soc., Providence, RI, 1997.

[3] P. Griffiths and J. Harris, Principles of algebraic geometry, Pure and Applied Mathematics, Wiley-Interscience, New York, 1978.

[4] N. J. Hitchin, Stable bundles and integrable systems, Duke Math. J. 54 (1987), 91-114.

[5] N. J. Hitchin, The moduli space of complex Lagrangian submanifolds, Asian Jour. Math. 3 (1999), 77-91.

[6] R. C. McLean, Deformations of calibrated submanifolds, Comm. Anal. Geom. 6 (1998), 705-747.

[7] S. Mukai, Symplectic structure of the moduli space of sheaves on an abelian or K3 surface, Invent. Math. 77 (1984), 101-116.

[8] C. Voisin, Sur la stabilité des sous-variétés lagrangiennes des variétés symplectiques holomorphes, Complex projective geometry (Trieste, 1989/Bergen, 1989), 294-303, London Math. Soc. Lecture Note Ser., 179, Cambridge Univ. Press, Cambridge, 1992.

[9] K. Yano and S, Bochner, Curvature and Betti numbers, Annals of Mathematics Studies, No. 32, Princeton University Press, Princeton, N. J., 1953.

[10] S.-T. Yau, On the Ricci curvature of a compact Kähler manifold and the complex Monge-Ampère equation. I, Comm. Pure Appl. Math. 31 (1978), 339-411. 